\title{
Função sensorial em membros superiores e atividades funcionais de indivíduos pós AVC
}

\section{Sensory function in upper limb and functional activities of individuals after stroke}

\section{Jamine Cunha dos Reis ${ }^{1}$, Maria Soraida Silva $\mathrm{Cruz}^{2}$, Ada Salvetti Cavalcanti Caldas ${ }^{3}$}

http://dx.doi.org/10.11606/issn.2238-6149.v31i1-3p46-53

Reis JC, Cruz MSS, Caldas ASC. Função sensorial em membros superiores e atividades funcionais de indivíduos pós AVC. Rev Ter Ocup Univ São Paulo. 2020 jan.-dez.;31(1-3):46-53.

RESUMO: O Acidente Vascular Cerebral é uma patologia incapacitante e tem alto índice de morbimortalidade no Brasil. Essa condição pode gerar um impacto na homeostase das funções motoras e sensoriais. São necessários mais estudos de reabilitação com essa população visto que há lacunas no que se refere à descrição das intervenções e orientações específicas para a função sensorial. Diante disto, objetivou-se relacionar a função sensorial com a função motora e a capacidade funcional de indivíduos após AVC. Foram utilizadas a Avaliação Sensorial de Nottingham, Medida de Independência Funcional e Escala Fugl Meyer. A amostra foi composta por 35 voluntários sendo observados que quanto maior a pontuação na função sensorial melhor seria o desempenho nas funções motoras. Ao associar a função sensorial, o desempenho motor e as atividades funcionais os sujeitos necessitavam de supervisão; foram mais independentes os que apresentaram os itens "toque" e "pressão" considerados como normais. Conclui-se que houve alterações das funções somatossensoriais e motoras que geraram impacto nas atividades funcionais.

PALAVRAS-CHAVE: Extremidade superior; Sensação; Acidente vascular cerebral; Reabilitação; Atividade motora.
Reis JC, Cruz MSS, Caldas ASC. Sensory function in upper limb and functional activities of individuals after stroke. Rev Ter Ocup Univ São Paulo. 2020 Jan-Dec;31(1-3):46-53.

ABSTRACT: Stroke is a disabling disease and has a high rate of morbidity and mortality in Brazil. This condition can have an impact on the homeostasis of motor and sensory functions. Further rehabilitation studies are needed with this population, as there are gap regarding the description of interventions and specific guidelines for sensory function. Given this, the objective was to relate the sensory function with the motor function and the functional capacity of individuals after stroke. Nottingham Sensory Assessment, Functional Independence Measure and Fugl Meyer Scale were used. The sample consisted of 35 volunteers and it was observed that the higher the score on sensory function, the better the performance on motor functions. When associating sensory function, motor performance and functional activities, the subjects needed supervision; those who presented the items "touch" and "pressure" were considered more independent. It was concluded that there were changes in somatosensory and motor functions that impacted functional activities.

KEYWORDS: Upper extremity; Sensation; Stroke; Reabilitation; Motor activity.

Este estudo foi parte integrante da conclusão do Programa de Residência de Reabilitação Física, do Instituto de Medicina Integral Professor Fernando Figueira

1. Terapeuta Ocupacional- Universidade Federal de Pernambuco; Especialização em Reabilitação Física - Instituto de Medicina Integral Professor Fernando Figueira- Recife (PE), Brasil. ORCID: 0000-0002-7259-8713. Email: jamine.c1@gmail.com

2. Terapeuta Ocupacional e Mestre em Saúde da Criança e do Adolescente na Universidade Federal de Pernambuco- Recife (PE), Brasil. ORCID:0000-0001-6894-9536. Email:soraida.to@gmail.com

3. Terapeuta Ocupacional e Doutora em Neuropsiquiatria e Ciências do Comportamento na Universidade Federal de Pernambuco - Recife (PE), Brasil.ORCID:0000-0002-6862-3728. Email: adasc@hotmail.com

Endereço para correspondência: Janine Cunha dos Reis. Rua Leonardo da Vinci, apartamento 205, bloco A, Jaboatão dos Guararapes, Pernambuco. 
Reis JC, et al. Função sensorial em membros superiores. Rev Ter Ocup Univ São Paulo. 2020 jan.-dez.;31(1-3):46-53.

\section{INTRODUÇÃO}

$\mathrm{O}^{2}$ Acidente Vascular Cerebral é uma patologia pode ser incapacitante e tem alto índice de morbimortalidade no Brasil, de acordo com as Diretrizes de Atenção à Reabilitação da Pessoa com Acidente Vascular Cerebral $^{1}$. O AVC tem maior prevalência em sujeitos idosos, do sexo masculino, com menor escolaridade, e moradores de áreas urbanas ${ }^{2}$.

Como consequência, os prejuízos decorrentes do AVC podem gerar impacto na homeostase das funções motoras $\mathrm{e}$ sensoriais dependendo da extensão da lesão, área e artéria cerebrais comprometidas pela hemorragia ou isquemia, visto que afetará regiões e vias de controle central. Entre essas vias estão às responsáveis pela condução e retroalimentação dos estímulos sensoriais captados do ambiente e do próprio corpo para orientar, regular e modular os mais diversos movimentos ${ }^{3}$.

Para auxiliar o indivíduo na compreensão da posição, percepção do corpo e interação com o ambiente, o sistema somatossensorial é formado por receptores sensoriais específicos para cada sensação incluindo o toque, dor, temperatura e a propriocepção ${ }^{3}$. Quando a informação sensorial não consegue ser adequadamente recebida e processada pelo Sistema Nervoso Central (SNC), o sujeito pode apresentar dificuldades e até a não realização devido aos comprometimentos da sensibilidade no(s) membro(s) comprometido(s), tais como a hipoestesia ${ }^{4}$.

Os comprometimentos motores incluem a alteração de força e amplitude de movimento; e quando acompanhados a prejuízos das funções somatossensoriais táteis e proprioceptivas acarretam em alterações dos padrões sinérgicos no movimento nos Membros Superiores (MMSS) podendo gerar prejuízos a capacidade funcional do sujeito5. Dessa forma, diversos são os impactos às atividades funcionais, tais como dependência no vestir, alimentar-se e escovar os dentes ${ }^{5}$.

Doyleet al. ${ }^{5}$ realizaram estudo no qual foram avaliados o discurso dos participantes quanto a percepção dos membros superiores nos aspectos função sensorial, deficiências sensoriais, sua recuperação e reabilitação ${ }^{5}$. Os autores observaram que os déficits sensoriais causaram impacto negativo no desempenho das atividades funcionais, e seu impacto leva o sujeito podendo a distanciar-se de atividades, com principalmente as que são realizados fora do domicílio, tais como: lazer e trabalho. Os sujeitos também descreveram o quanto é importante o suporte e rede de apoio dos familiares e profissionais de saúde neste processo de adaptação.

Scalha et al. ${ }^{6}$ pesquisaram 20 pacientes com sequelas de $\mathrm{AVC}$, identificaram que todos os participantes apresentaram dificuldade nas modalidades somatossensoriais e comprometimento motor (tais como hemiplegia) principalmente com pior desempenho nos itens avaliados com oclusão da visão nas escalas Fugl Meyer e Avaliação sensorial de Nottingham ${ }^{6}$. Constatou-se ainda o prejuízo da função sensorial desencadeou mais dificuldades nas funções motoras em membros superiores o que causou impacto negativo nas atividades funcionais.

Nesse contexto, a literatura aponta que existe relação entre as atividades funcionais, função sensorial e funções motoras. Porém, valem ressaltar a necessidade mais estudos para o embasamento para a prática clínica de profissionais de reabilitação envolvidos com essa população visto que ainda há uma lacuna no que se refere à descrição das intervenções e orientações específicas para a função sensorial. O estudo tem por objetivo relacionar a função sensorial com a função motora e a capacidade funcional de indivíduos após AVC.

\section{PROCEDIMENTOS METODOLÓGICOS}

Foi realizado um estudo de natureza quantitativa, do tipo observacional, de corte transversal em um Centro Especializado em Reabilitação Nível IV na cidade do Recife, em Pernambuco. O período de coleta foi de agosto de 2018 a fevereiro de 2019. Na amostra foram incluídos pacientes com sequelas de AVC; atendidos no local, de ambos os sexos com idade igual e/ou acima de dezoito anos com dificuldade de movimentação de membro(s) superior(es) e sem dificuldades cognitivas para compreender o questionário de avaliação ou falado pelo pesquisador. Foram excluídos os pacientes com sequelas decorrentes de outras patologias neurológicas e/ou osteomusculares.

Para a coleta de dados inicialmente foi realizado o levantamento dos pacientes atendidos na instituição. Foram verificados os critérios de elegibilidade, em seguida os pacientes foram convidados a participar da pesquisa nos dias em que frequentaram o serviço, sem prejuízo nas suas terapias regulares, no qual foi feita uma listagem em planilha do Excel dos participantes.

Foram aplicados os seguintes instrumentos: questionário de características sociodemográficas e de condições clínicas; Escala de Avaliação Fugl Meyer (EFM) no qual foram avaliados apenas os itens para membros superiores: Sensibilidade/extereocepção, propriocepção, sinergia extensora e flexora, motricidade reflexa, movimentos com e sem sinergias, controle de punho e mão totalizando a pontuação 66 , considerando que cada item apresenta pontuação de 0 a 2 , sendo ( 0 alterado, 1 parcialmente e 2 totalmente alterado $)^{7}$; Avaliação Sensorial de Nottingham (ASN) nos quais foram considerados os itens: Presença de 
Reis JC, et al. Função sensorial em membros superiores. Rev Ter Ocup Univ São Paulo. 2020 jan.-dez.;31(1-3):46-53.

edema, toque, pressão, picada, temperatura, localização, toque bilateral, propriocepção, estereognosia, discriminação de dois pontos na mão e dedos, sendo classificados como: normal (pontuação 2) e alterado (pontuação 0 ou 1: sendo o 0 não apresentou nenhuma resposta, e 1 quando modificado) ${ }^{8}$; e a Medida de Independência Funcional (MIF) no qual os indivíduos foram classificados como independentes (pontuação total de 91 a 126) e dependentes (pontuação total de 18 a 90$)^{9}$. Foram realizadas as médias e desvio padrão da pontuação total das avaliações MIF e EFM.

Para as análises dos dados foram utilizados os Softwares STATA/SE 12.0 e o Excel 2010. As variáveis numéricas estão representadas pelas medidas de tendência central e medidas de dispersão. Para comparação das variáveis quantitativas utilizou-se o teste Qui-Quadrado e o Teste Exato de Fisher; o Teste de Normalidade dos dados foram determinados usando o teste de Kolmogorov-Smirnov para variáveis quantitativas; e para comparação com dois grupos o Teste t Student (para a Distribuição Normal). Todos os testes foram aplicados com 95\% de confiança e apresentados em forma de tabela com suas respectivas frequências absoluta e relativa.

O projeto foi submetido ao Comitê de Ética em Pesquisa Envolvendo Seres Humanos de acordo com a Resolução $n^{\circ}$ 466/12 do Conselho Nacional de Saúde (CNS), sendo o número de aprovação foi 93318418.9.0000.5201. A coleta de dados foi iniciada após a aprovação do projeto de pesquisa pelo Comitê de Ética em Pesquisa e após a assinatura do Termo de Consentimento Livre e Esclarecido por parte de todos os sujeitos.

\section{RESULTADOS}

A amostra foi composta por 35 voluntários com média de idade de 57,4 anos (desvio padrão: $\pm 11,57$ ) sendo 23 homens e 12 mulheres. Dentre os tipos de AVC, o de maior predomínio foi o isquêmico com tempo de lesão de mais de um ano. Foi observado o maior acometimento do hemicorpo esquerdo $(65,7 \%)$; todos os pacientes com dominância do lado direito (100\%); sem presença de edema no membro (94,3\%), adultos, no estado civil: casado ou em união estável $(65,7 \%)$, com renda familiar menor que um salário mínimo $(48,5 \%)$, tendo como principal comorbidade a Hipertensão Arterial Sistêmica (91,4\%), conforme descrito na Tabela 1.

No Gráfico 1 foram descritas as pontuações da ASN, organizando os itens em ordem decrescente com relação ao comprometimento sensorial. Desta forma, destacou-se o item estereognosia $(91,4 \%)$ como o de maior déficit, enquanto a percepção da pressão $(34,3 \%)$ foi a menos comprometida.
Tabela 1 - Descrição das características sociodemográficas e de condições clínicas $(\mathrm{n}=35)$

\begin{tabular}{|c|c|c|}
\hline Variáveis & $\begin{array}{r}\text { Frequência } \\
\text { absoluta (n) }\end{array}$ & $\begin{array}{l}\text { Frequência } \\
\text { relativa(\%) }\end{array}$ \\
\hline \multicolumn{3}{|l|}{ Idade } \\
\hline 30 a 40 anos & 1 & 2,9 \\
\hline 41 a 50 anos & 10 & 28,6 \\
\hline 51 a 60 anos & 10 & 28,6 \\
\hline 61 a 70 anos & 9 & 25,6 \\
\hline 71 a 80 anos & 5 & 14,3 \\
\hline \multicolumn{3}{|l|}{ Sexo } \\
\hline Feminino & 12 & 34,3 \\
\hline Masculino & 23 & 65,7 \\
\hline \multicolumn{3}{|l|}{ Arranjo familiar } \\
\hline Solteiro(a) & 10 & 28,6 \\
\hline Casado(a) ou união estável & 23 & 65,7 \\
\hline Viúvo(a) & 2 & 5,7 \\
\hline \multicolumn{3}{|l|}{ Renda familiar (SM) } \\
\hline Menos de um SM & 17 & 48,5 \\
\hline Um SM & 14 & 40,0 \\
\hline Dois SM & 3 & 8,6 \\
\hline Três ou mais SM & 1 & 2,9 \\
\hline \multicolumn{3}{|l|}{ Tempo de lesão } \\
\hline Menos de um ano de lesão & 5 & 14,3 \\
\hline Um ano de lesão & 11 & 31,4 \\
\hline Mais de um ano de lesão & 19 & 54,3 \\
\hline \multicolumn{3}{|l|}{ Tipo de AVC } \\
\hline Isquêmico & 29 & 82,9 \\
\hline Hemorrágico & 6 & 17,1 \\
\hline \multicolumn{3}{|l|}{ Comorbidades } \\
\hline HAS & 32 & 91,4 \\
\hline $\mathrm{DM}$ & 9 & 25,7 \\
\hline Outras & 8 & 22,9 \\
\hline \multicolumn{3}{|l|}{ Mão dominante } \\
\hline Direita & 35 & 100,0 \\
\hline Esquerda & 0 & 0,0 \\
\hline \multicolumn{3}{|l|}{ Lado acometido } \\
\hline Direita & 12 & 34,3 \\
\hline Esquerda & 23 & 65,7 \\
\hline \multicolumn{3}{|l|}{ Presença de edema } \\
\hline Sim & 2 & 5,7 \\
\hline Não & 33 & 94,3 \\
\hline
\end{tabular}

*AVC= Acidente Vascular Cerebral; SM= Salário Mínimo; HAS= Hipertensão Arterial Sistêmica; DM= Diabetes Mellitus 
Gráfico 1- Descrição dos itens da Avaliação Sensorial de Nottingham na ordem do mais comprometido ao menos comprometido

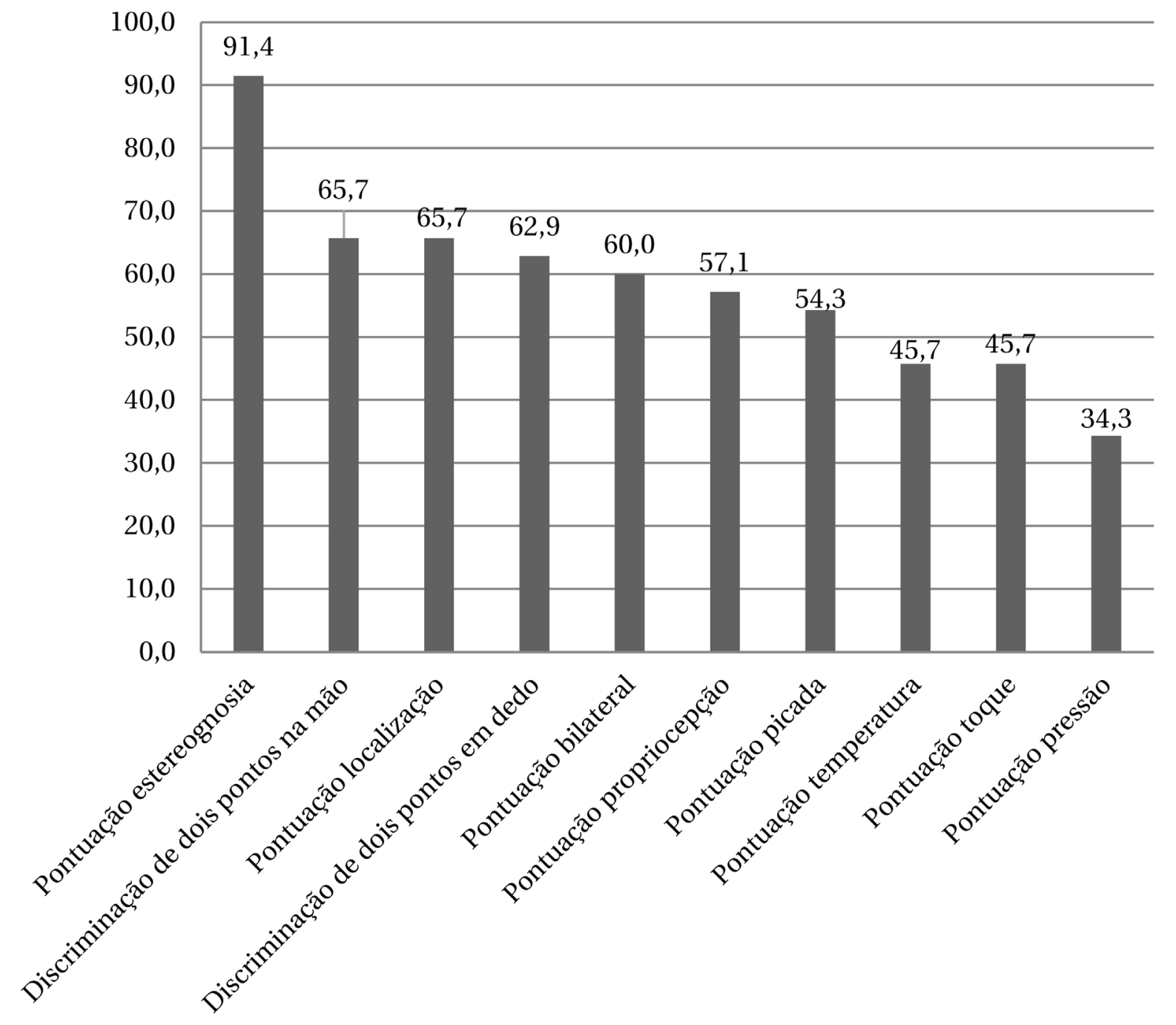

Nas avaliações funcionais, os participantes obtiveram a média de 96,7 pontos (desvio padrão: $\pm 18,33$ ) na MIF e 38,08 pontos (desvio padrão: $\pm 20,63$ ) na EFM. Na MIF, destaca-se a ocorrência de 11 voluntários $(31,5 \%)$ com a classificação de independência modificada, ou seja, não necessitavam de ajuda para realização de suas atividades diárias, mas realizavam-nas com lentidão e/ou com instrumentais (órteses, adaptações dentre outros). Já 14 sujeitos $(40 \%)$ precisam de supervisão para realização das atividades do cotidiano, enquanto 07 (20\%) realizaram suas atividades com assistência mínima e 03 (8,5\%) com assistência moderada.
Com base na Tabela 2, na associação dos domínios de membro superior das Escalas de Avaliação de Nottingham e da Fugl Meyer, observa-se que houve diferença estatisticamente significante nas variáveis: toque, pressão, picada, localização, toque bilateral, propriocepção e discriminação de dois pontos nos dedos. Dessa forma, é possível supor que quanto mais preservada a função sensorial no membro, melhor será a função motora.

Observa-se na Tabela 3 que a maior parte dos sujeitos classificados como dependentes avaliados pela MIF também tiveram a pontuação alterada, em frequência absoluta, para as seguintes funções sensoriais: localização, toque bilateral e discriminação de dois pontos em mão e dedos. 
Reis JC, et al. Função sensorial em membros superiores. Rev Ter Ocup Univ São Paulo. 2020 jan.-dez.;31(1-3):46-53.

Tabela 2 - Associação das medidas de domínios das avaliações Escala Sensorial de Nottingham e Fugl Meyer com média e desvio padrão $(\mathrm{n}=35)$

\begin{tabular}{|c|c|c|}
\hline \multirow[t]{2}{*}{ Variáveis } & $\begin{array}{l}\text { Pontuação Total } \\
\text { Fugl Meyer }\end{array}$ & \multirow[t]{2}{*}{ p-valor* } \\
\hline & Média \pm DP & \\
\hline \multicolumn{3}{|l|}{ Toque } \\
\hline Alterado & $29,25 \pm 22,72$ & 0,024 \\
\hline Normal & $45,53 \pm 16,44$ & \\
\hline \multicolumn{3}{|l|}{ Pressão } \\
\hline Alterado & $25,92 \pm 24,42$ & 0,030 \\
\hline Normal & $44,43 \pm 16,01$ & \\
\hline \multicolumn{3}{|l|}{ Picada } \\
\hline Alterado & $30,32 \pm 22,61$ & 0,012 \\
\hline Normal & $47,31 \pm 14,59$ & \\
\hline \multicolumn{3}{|l|}{ Temperatura } \\
\hline Alterado & $30,63 \pm 21,68$ & 0,052 \\
\hline Normal & $44,37 \pm 18,58$ & \\
\hline \multicolumn{3}{|l|}{ Localização } \\
\hline Alterado & $32,22 \pm 21,38$ & 0,019 \\
\hline Normal & $49,33 \pm 15,20$ & \\
\hline \multicolumn{3}{|c|}{ Toquebilateral } \\
\hline Alterado & $32,29 \pm 21,79$ & 0,043 \\
\hline Normal & $46,79 \pm 16,73$ & \\
\hline \multicolumn{3}{|c|}{ Propriocepção } \\
\hline Alterado & $30,30 \pm 21,45$ & 0,009 \\
\hline Normal & $48,47 \pm 15,44$ & \\
\hline \multicolumn{3}{|c|}{ Estereognosia } \\
\hline Alterado & $36,34 \pm 20,63$ & 0,109 \\
\hline Normal & $56,67 \pm 17,04$ & \\
\hline \multicolumn{3}{|c|}{$\begin{array}{l}\text { Discriminação de } \\
\text { dois pontos na mão }\end{array}$} \\
\hline Alterado & $34,39 \pm 22,98$ & 0,102 \\
\hline Normal & $45,17 \pm 14,70$ & \\
\hline \multicolumn{3}{|c|}{$\begin{array}{l}\text { Discriminação de } \\
\text { dois pontos em dedo }\end{array}$} \\
\hline Alterado & $32,64 \pm 22,57$ & 0,025 \\
\hline Normal & $47,31 \pm 14,30$ & \\
\hline
\end{tabular}

(*) Teste t Student
Tabela 3 - Comparação entre as variáveis de atividades funcionais da Medida de Independência Funcional com a função sensoriomotora das avaliações Sensorial de Nottingham e Fugl Meyer.

\begin{tabular}{|c|c|c|c|}
\hline \multirow{3}{*}{ Variáveis } & \multicolumn{2}{|c|}{ Pontuação MIF Total } & \multirow{3}{*}{ p-valor } \\
\hline & Dependência & Independência & \\
\hline & n (\%) & n (\%) & \\
\hline \multicolumn{4}{|l|}{ Toque } \\
\hline Alterado & $11(68,8)$ & $5(31,2)$ & $0,983 *$ \\
\hline Normal & $13(67,4)$ & $6(31,6)$ & \\
\hline \multicolumn{4}{|l|}{ Pressão } \\
\hline Alterado & $8(66,7)$ & $4(33,3)$ & $1,000 * *$ \\
\hline Normal & $16(69,6)$ & $7(30,4)$ & \\
\hline \multicolumn{4}{|l|}{ Picada } \\
\hline Alterado & $14(73,7)$ & $5(26,3)$ & $0,478 *$ \\
\hline Normal & $10(62,5)$ & $6(37,5)$ & \\
\hline \multicolumn{4}{|l|}{ Temperatura } \\
\hline Alterado & $12(75,0)$ & $4(25,0)$ & $0,452 *$ \\
\hline Normal & $12(63,2)$ & $7(36,8)$ & \\
\hline \multicolumn{4}{|l|}{ Localização } \\
\hline Alterado & $16(69,6)$ & $7(30,4)$ & $1,000 * *$ \\
\hline Normal & $8(66,7)$ & $4(33,3)$ & \\
\hline \multicolumn{4}{|c|}{ Toque bilateral } \\
\hline Alterado & $16(76,2)$ & $5(23,8)$ & $0,283 * *$ \\
\hline Normal & $8(57,1)$ & $6(42,9)$ & \\
\hline \multicolumn{4}{|c|}{ Propriocepção } \\
\hline Alterado & $15(75,0)$ & $5(25,0)$ & $0,467 * *$ \\
\hline Normal & $9(60,0)$ & $6(40,0)$ & \\
\hline \multicolumn{4}{|c|}{ Estereognosia } \\
\hline Alterado & $21(65,6)$ & $11(34,4)$ & $0,536^{* *}$ \\
\hline Normal & $3(100,0)$ & $0(0,0)$ & \\
\hline \multicolumn{4}{|c|}{$\begin{array}{l}\text { Discriminação de } \\
\text { dois pontos na mão }\end{array}$} \\
\hline Alterado & $16(69,6)$ & $7(30,4)$ & $1,000 * *$ \\
\hline Normal & $8(66,7)$ & $4(33,3)$ & \\
\hline \multicolumn{4}{|c|}{$\begin{array}{l}\text { Discriminação de } \\
\text { dois pontos em dedo }\end{array}$} \\
\hline Alterado & $16(72,7)$ & $6(27,3)$ & $0,708 * *$ \\
\hline Normal & $8(61,5)$ & $5(45,5)$ & \\
\hline
\end{tabular}

(*) Teste Qui-Quadrado (**) Teste Exato de Fisher

\section{DISCUSSÃO}

Os resultados do estudo mostram que quanto aos dados clínicos e sociodemográficos, o AVC é muito associado 
Reis JC, et al. Função sensorial em membros superiores. Rev Ter Ocup Univ São Paulo. 2020 jan.-dez.;31(1-3):46-53.

à população idosa, porém esta patologia tem cada vez mais afetado indivíduos adultos. Corroborando com o que foi avaliado neste trabalho, a maioria dos pacientes tem idades de 41 a 60 anos $^{2}$. Podendo trazer impacto para a economia, visto que são pessoas em idades produtivas que ficam afastadas do mercado de trabalho.

Em relação ao sexo verificou-se que o número de homens é superior ao de mulheres. Acredita-se que estes buscam menos os serviços de atenção voltados a prevenção das doenças e promoção à saúde e acabam por necessitar mais da reabilitação, devido às sequelas decorrentes de diversas patologias. Também se destaca a hipertensão arterial sistêmica como mais prevalente, sendo uma das principais comorbidades que levam ao AVC; e que dentre os tipos de AVC o isquêmico foi mais predominante o que ratifica com a literatura, pois muitos hemorrágicos levam a morte em $80 \%$ dos casos ${ }^{2}$.

As rendas familiares foram em sua maioria baixa, com valor menor que o salário mínimo per capta, o que pode dificultar o acesso a serviços de saúde e oportunidades de cuidado, visto que as necessidades básicas do sujeito são suprimidas. Quanto ao arranjo familiar, a maioria dos pacientes estava casado ou apresentaram união estável, dado relevante, visto que representa maior rede de apoio e cuidados. Anderson e colaboradores realizaram estudo que retratou o relacionamento conjugal após o $\mathrm{AVC}^{10}$. Observaram que a qualidade do vínculo pode influenciar na recuperação, dificuldades nos cuidados e qualidade de vida. A partir relatos dos sujeitos da pesquisa, foi possível analisar que todos os pacientes mesmo os que se separam após o AVC atribuíam o seu bem estar aos cuidados recebidos pelo cônjuge.

A descrição das funções sensoriais e motoras, proposta por este estudo, revelou que em todos os indivíduos os itens da ASN que apresentaram pontuação normal foram associados à maior pontuação motora pela EFM. Permitenos observar que quanto mais superiora pontuação da função sensorial melhor será o desempenho nas funções motoras e como consequência podemos inferir os impactos nas atividades funcionais. Como mostra no estudo de Carey et al. ${ }^{4}$ realizado com 268 pacientes pós AVC, observaram que o déficit somatossensorial em membros superiores e inferiores, mesmo sem a perda da função motora causam impacto na restrição das atividades funcionais ${ }^{4}$.

Ao analisar os itens específicos da ASN, o toque teve e a propriocepção obtiveram relevância estatisticamente significante quanto à associação das funções motoras com a EFM. Esse resultado se aproxima do descrito por Meyer e colaboradores onde realizaram a associação da propriocepção com o toque leve em pacientes com $\mathrm{AVC}$, no qual indicou significativa repercussão quanto à recuperação motora dos membros superiores. Os autores observaram que quando essas funções estavam prejudicadas, os sujeitos apresentaram dificuldades e impactos negativos nas atividades de vida diária e papéis sociais ${ }^{11}$.

Ainda em concordância com os resultados da presente pesquisa, Cherpin et al. ${ }^{12}$, investigaram a correlação dos déficits motores com a propriocepção em indivíduos saudáveis e indivíduos pós $\mathrm{AVC}^{12}$. Eles observaram que $40 \%$ dos sujeitos que sofreram a patologia apresentou alteração proprioceptiva, e quanto maior o comprometimento, menor a função motora, visto que este item auxilia diretamente no controle e planejamento motor ${ }^{12}$.

Em relação à discriminação de dois pontos em mão e dedos os resultados apontaram que os sujeitos com maior pontuação somatossensorial também obtiveram maior pontuação na função motora, Corroborando com esse estudo Meyer et al. ${ }^{11}$ realizaram uma revisão sistemática na literatura com o objetivo de identificar os comprometimentos somatossensoriais com os motores nos membros superiores de indivíduos após o $\mathrm{AVC}^{11}$. Dentre as pesquisas identificadas, seis relataram a influência dos comprometimentos somatossensoriais na função motora, atividade e participação; e que dentre os componentes somatossensoriais a recuperação da discriminação de dois pontos foi um bom preditor na reabilitação dos membros superiores tendo repercussões no cotidiano do sujeito ${ }^{11}$.

Quando analisamos em termos de frequência o comprometimento sensorial, foi observado que a estereognosia foi a mais presente. Nesse sentido, o estudo de Connell et al. ${ }^{14}$ também buscou investigar a frequência do comprometimento somatossensorial em pacientes com AVC agudo em diferentes modalidades somatossensoriais e diferentes áreas do corpo, e sua recuperação ${ }^{14}$. Observaram que houve maior impacto na sensação tátil e na estereognosia, com esta última sendo a mais frequente, corroborando com o estudo atual. Também relataram que $17 \%$ dos participantes apresentaram comprometimento sensorial do lado contralateral a hemiparesia ${ }^{14}$.

Outro aspecto relevante nesse estudo foi que a maior parte da população estudada, quanto ao nível de dependência nas atividades funcionais, foi classificada como necessitando de "supervisão ou preparação", ou seja, demandam da presença de terceiros ou instruções sem necessidade de toque físico. Os autores acreditam que esta pontuação pode ser decorrente da maioria dos pacientes ter tido o lado esquerdo acometido, e todos têm a mão dominante direita, sem presença de edema, então, possivelmente, os sujeitos desenvolveram estratégias (pessoais em domicílio e adquiridas no processo de reabilitação) que facilitam o seu desempenho9. Isto é um fator que se torna facilitador no retorno das atividades funcionais. 
Reis JC, et al. Função sensorial em membros superiores. Rev Ter Ocup Univ São Paulo. 2020 jan.-dez.;31(1-3):46-53.

Como visto, as funções sensoriomotoras também são um fator que podem influenciar o desempenho dos sujeitos pós AVC em sua rotina. Nossos resultados sugerem que os sujeitos que obtiveram descrição como "normal" na ASN possuíram maior pontuação na MIF em relação aos que apresentaram a função "alterada". Essas funções são importantes em atividades como vestir as roupas e amarrar o sapato já que os itens auxiliam na graduação de força e coordenação.

Um relevante resultado observado foi a estereognosia como o item de maior pontuação prejudicado nos participantes, onde os sujeitos eram mais dependentes à medida que este item era alterado. Resultados semelhantes foram encontrados no estudo de Cruz et al. ${ }^{13}$, que investigaram a associação entre os componentes de sensibilidade para estereognosia, função manual e a independência nas atividades de vida diária em indivíduos com sequelas de AVC. Foi encontrado que a maior pontuação da estereognosia teve associação com o maior nível de independência com a mão menos comprometida (lado contralateral).

\section{CONCLUSÃO}

Como contribuição para a prática clínica, inferimos que houve alterações das funções somatossensoriais e motas que geraram impacto nas atividades funcionais. Observamos que a maioria dos pacientes necessitou de supervisão nas atividades funcionais, e necessitaram da ajuda de terceiros os sujeitos que apresentaram os itens toque e pressão considerados como normais.

Como pontos fortes destacam-se o uso dos instrumentos de avaliação utilizados, visto que são de boa qualidade, largamente utilizado na literatura, de baixo custo, podem ser utilizados no Sistema Único de Saúde, de fácil aplicação e aplicabilidade na prática clínica. Foram encontradas como limitações do estudo a quantidade de sujeitos e o tempo de coleta. Sugere-se para próximos trabalhos, o aumento do número de participantes e do tempo de coleta para que tenha maior representatividade na população geral, podendo ser ampliado para outros serviços.

Participação dos autores: Todas as autoras referidas colaboraram no processo de concepção e o delineamento ou a anális

\section{REFERÊNCIAS}

1. Brasil. Ministério da Saúde. Secretaria de Atenção à Saúde. Departamento de Ações Programáticas Estratégicas. Diretrizes de atenção à reabilitação da pessoa com acidente vascular cerebral. Brasília: Ministério da Saúde; 2013.

2. Bensenor IM, Goulart AC, Szwarcwald CL, Vieira MLFP, Malta DC, Lotufo PA. Prevalence of stroke and associate disability in Brazil: National Health Survey - 2013. Arq Neuro-Psiquiatr. 2015;73(9):746-50. DOI : 10.1590/0004-282x20150115.

3. Borstad AL, Nichols-LarsenDS. Assessing and Treating Higher Level Somatosensory Impairments Post Stroke. Topics Stroke Rehabil. 2014;21(4), 290-5. DOI: 10.1310/tsr2104-290.

4. Carey LM, Matyas T A,Baum C. Effects of Somatosensory Impairment on Participation After Stroke. Am J Occup Ther. 2018;72(3):1220-31. DOI: 10.5014/ajot.2018.025114.

5. Doyle SD, Bennett S, Dudgeon B. Upperlimb post-stroke sensory impairments: the survivor'sexperience. Disabil Rehabil. 2013;36(12):993-1000. DOI: 10.3109/09638288.2013.825649.

6. Scalha ITB,Miyasaki E, Lima NMFV, Borges G. Correlations between motor and sensory functions in upper limb chronic hemiparéticos after stroke. Arq Neuro-Psiquiatr. 2011;69(4):624-9. DOI: 10.1590/S0004-282X2011000500010.

7. Padovani C, Pires CVG, Ferreira FPC, BoreinG, Filippo TRM, Imamura M. Aplicação das escalas Fugl-Meyer Assessment
(FMA) e Wolf Motor Function Test (WMFT) na recuperação funcional do membro superior em pacientes pós-acidente vascular encefálico crônico: revisão de literatura. Acta Fisiatr. 2013;20(1):42-9.DOI:10.5935/0104-7795.20130008.

8. Lima DF, Queiroz AP, Salvo D, Yoneyama SM, Oberg TD, Lima MFV. Versão brasileira da avaliação sensorial de Nottingham: validade, concordância e confiabilidade. Rev Bras Fisioter. 2010;14(2):166-74. DOI: 10.1590/S1413-35552010005000006.

9. Riberto M, Miyazaki MH, Jucá SSH, Sakamoto H, Pinto PPN, Battistella LR. Validação da versão brasileira da Medida de Independência Funcional. Acta Fisiatr. 2004;11(2):72-6. DOI: 10.5935/0104-7795.20040003.

10. Anderson S, Keating N, Wilson D. Reconciling Marriage and Care after Stroke. Can J Aging. 2017;36(03):386-401. DOI: $10.1017 / \mathrm{s} 0714980817000241$.

11. Meyer S, Karttunen AH, Thijs V, Feys H, Verheyden G. How do somatosensory deficits in the armand hand relate to upper limb impairment, activity, and participation problems after stroke? A Systematic Review. PhysTher. 2014;94(9):122031. DOI: $10.2522 / \mathrm{ptj} .20130271$.

12. Cherpin A, Kager S, Budhota A, Contu S, Vishwanath D, Kuah CW, et al. A preliminary study on th erelation ship 
Reis JC, et al. Função sensorial em membros superiores. Rev Ter Ocup Univ São Paulo. 2020 jan.-dez.;31(1-3):46-53.

between proprioceptive deficits and motor functions in chronic stroke patients. IEEE Int Conf Rehabil Robot. 2019;465-470. DOI: 10.1109/ICORR.2019.8779447.

13. Cruz DMC, Silva NS, Patti LP, Paiva G, Paolillo AR. Correlação entre sensibilidade, função manual e independência em indivíduos pós acidente vascular cerebral. Rev Paraense
Med. 2015;29(1). Disponível em: http://files.bvs.br/ upload/S/0101-5907/2015/v29n1/a4654.pdf

14. Connell LA, Lincoln NB, Radford KA.Somatosensory impairment after stroke: frequency of different deficits and the irrecovery. Clin Rehabil. 2008;22(8):758-67. DOI: $10.1177 / 0269215508090674$. 